ANNALS (IF PHYSICS: 3, 275-291 (1958)

\title{
The Study of Nuclear Collective Motion by Stripping Reactions*
}

\author{
G. R. SaTCHLER \\ Department of Physirs, University of Mirhigan, Ann Arbor, Wichigan
}

\begin{abstract}
The 'intrinsic' probability for capture of nucleons is studied for nuclei which exhibit collective vibrations or rotations in addition to an underlying shell structure of independent nucleon orbits. Such probabilities or reduced widths for low-lying nuclear levels can be measured in 'direct' nuclear reactions; the present discussion is formulated chiefly in terms of deuteron stripping. Various selection rules are derived, and possible reasons for their breakdown studied. It is found that stripping is a particularly direct way of studying the wave functions of single particle orbits in strongly deformed nuclei. There are simple branching ratios between the cupture probabilities into the various members of a rotational band in such nuclei. Finally, it is shown how such reduced width measurements can provide an independent check on the wave functions postulated by Osborn and Kilema to correlate empirically the ground state properties of nuclei.
\end{abstract}

\section{INTRODUCTION}

In a deuteron stripping reaction (1), the rôle of the incident deuteron is to present at the target nucleus surface a neutron or proton ready to be captured (or 'stripped' off). Since its partner in the deuteron is available to carry off any residual energy, this allows capture directly into negative energy, or bound, states, without the complications of an intermediate compound nucleus in which the whole deuteron is absorbed. In this way the stripping reaction provides a sensitive tool for studying the wave functions of such nuclear states through the measurement of their reduced widths, or intrinsic probabilities for nucleon capture. In many ways it is a less ambiguous tool than, for example, analysis of electromagnetic moments and transitions, for these involve additional assumptions concerning current densities, gyromagnetic ratios, etc. These remarks apply also to the stripping of other projectiles (such as in $\left(\alpha, \mathrm{He}^{3}\right.$ ) reactions) in which a nucleon is transferred to the target, or, of course, the inverse pickup reactions. While these reactions are not as well understood theoretieally as denteron stripping, the same nuclear overlap integral is involved as is

* This work was supported in part by the L. S. Atomic Energy Commission.

$\dagger$ ( )n leave from Clarendon Laboratory, Oxford, England. 
discussed in the next section. Similar factors occur in the description of other direct reactions ( 1 ) (Appendix I).

Such studies have been made for nuclei which are described well by the shell model (in ref. 2 for example). In the same way we can investigate the predictions of the nuclear collective model (3). Interest has been revived recently, particularly by the evidence for collective motion of the rotational type in lighter nuclei in the region of $\mathrm{Mg}(4)$ and even $\mathrm{F}^{19}$ (5). Here the Coulomb barrier does not obscure experiments with deuterons of moderate energy.

In this paper we study in detail the excitation of the recently identified nuclear vibrational spectra $(6)$ as well as stripping into rotational levels. In addition we consider the collective model of Osborn and Klema $(\gamma)$ which has been used to correlate the empirical properties of nuclear ground states. The aim is to present the theoretical predictions of these models rather than to analyze experimental data.

Recently, such analyses have been carried out by the Chalk River group (8) for the nuclei $\mathrm{Al}^{25}, \mathrm{Mg}^{25}$ and $\mathrm{Si}^{29}$ produced by deuteron stripping, in terms of collective rotations. The success of these should encourage further experimental work in this region.

The present work is, for the greater part, an elaboration of material presented in lectures at Washington University, St. Louis, in February, 1957. A preliminary study of this problem was made in an earlier publication $(9)$.

\section{STRIPPING ANI) NUCLEAR STRUCTURE}

To be definite we shall discuss the $(d, p)$ reaction. Apart from the absence of any Coulomb interaction with the outgoing particle, the description of a $(d, n)$ reaction is identical, and, of course, by reciprocity applies equally to the inverse pickup reactions.

The original Butler (10) and Born-approximation (11) expressions for the stripping reaction amplitude contain as a factor the overlap integral between the initial and final nuclear states. It also appears in the more realistic modified Born-approximation of Tobocman (12). In this, the incident deuteron waves and outgoing proton waves are allowed to be distorted by elastic scattering from suitable optical potentials' representing the target and residual nuclei, respectively. In fact this overlap factor will be a common feature of all treatments which neglect excitation of the target nucleus before capture and excitation of the final nucleus after capture ${ }^{1}$. The neglect of these excitations is in

1 Except for exchange contributions of the type considered by French (13) and called 'heavy particle stripping' by Owen and Madansky (14). These terms contain analogous overlap factors, but they are more complicated than those we discuss. In addition there may be small contributions from a coupling of the proton to the nuclear collective modes of motion. These are discussed in Appendix II. 
the spirit of the stripping picture. It seems unlikely they would show an angular distribution like the characteristic l-peaks of stripping, but rather provide a more-or-less isotropic background.

For the reaction $A(d, p) B$, this overlap factor may be written ${ }^{2}$

$$
\int d \xi \Psi_{I_{B} M_{B}}{ }^{*}\left(x_{N} \xi\right) \Psi_{I_{A} M_{A}}(\xi)=\Sigma_{j l} \beta_{j l} \phi_{j l m}\left(x_{N}\right)\left\langle I_{A} j M_{A} m \mid I_{B} M_{B}\right\rangle
$$

$x_{N}$ denotes the coordinates of the captured neutron, and $\xi$ those of the target nucleons. $\phi_{j l m}$ is a normalized spin-orbit function for the neutron, with total angular momentum $j$ and orbital $l . \Psi_{A}$ and $\Psi_{B}$ are the wave functions of the target and residual nuclear states, respectively. The expansion parameters $\beta_{j l}$ have an obvious physical interpretation. They measure the degree to which the residual nucleus $B$ can be regarded as made up of the target $A$ plus a neutron in an orbit with $j$ and $l$. That is, the degree to which $A$ is a parent state of $B(15)$. Thus in an extreme single-particle picture, only one $\beta$ will be nonvanishing, and that has the value unity. The last factor in (1), the Clebsch-Gordan or vector-addition coefficient (16), just gives the probability amplitude for finding the z-components $M_{A}$ and $m$ when their total is $M_{B}$, and is purely geometrical in content. Finally, in writing (1), by singling out the neutron labelled $x_{N}$ as being in the incident deuteron, we have explicitly neglected to antisymmetrise it with the other neutrons in the target nucleus. When we remedy this, if there are $n-1$ neutrons in nucleus $A$, we get $n$ terms like (1), each with a normalizing factor $1 / \sqrt{ } n$. So the total amplitude is $\sqrt{n}$ times the term containing (1), and the physically significant parameter is

$$
\theta_{j l}=\sqrt{n} \beta_{j l}
$$

Then $\theta_{j l}{ }^{2}$ is the reduced width in units of the so-called single particle reduced width $^{3}, \theta^{2}=\gamma^{2} / \gamma_{S P}{ }^{2}$.

When, as generally will be the case, the nuclear states are not described exactly by one simple model, the wave functions $\Psi_{A}, \Psi_{B}$ will be a certain mixture of the

$2 \beta$ and $\phi$ should really include a label $N$ for the principal quantum number of the orbit. However, the appearance in (1) of appreciable amounts of more than one $N$ for a given $j, l$ is unlikely. Moreover, they could not be distinguished experimentally. If more than one $N$ does contribute, we may interpret (1) in the following way : $\beta=\Sigma_{N} \beta_{N}, \phi=\Sigma_{N} \beta_{N} \phi_{N} / \beta$, so that $\phi$ is a weighted average.

${ }^{3}$ Occasionally the channel spin representation is used, where the channel spin $S$ is the vector sum of the spins of the target nucleus and captured nucleon, $S=I_{A}+\mathbf{s}$. The corresponding reduced widths $\theta_{S}$ are simply related to ours by the Racah change-of-coupling coeffieients (17)

$$
\theta_{S i}=\Sigma_{j} \theta_{j l} \sqrt{(2 S+1)(2 j+1)} W\left(I_{A}{ }^{1 / 2} I_{B} l ; S_{j}\right)^{j-i-1 / 2} .
$$


model wave functions, (for example, configuration mixing in the shell model),

$$
\begin{aligned}
& \Psi_{A}=\Sigma_{\alpha} K_{\alpha}(A) \Psi_{\alpha}, \\
& \Psi_{B}=\Sigma_{\beta} K_{\beta}(B) \Psi_{\beta} .
\end{aligned}
$$

The $\Psi_{\alpha}, \Psi_{\beta}$ are definite model states or configurations, and appear with amplitudes $K_{\alpha}, K_{\beta}$. Inserting these wave functions in the overlap integral (1) we have the reduced width amplitude expressed as a corresponding sum of terms

$$
\theta_{j l}=\Sigma_{\alpha \beta} K_{\alpha}(A) K_{\beta}(B)^{*} \theta_{j l}(\alpha \beta) .
$$

$\theta(\alpha \beta)$, of course, is obtained from the overlap of $\Psi_{\alpha}, \Psi_{B}$. It is important to note that the $\theta(\alpha \beta)$ for a given $j$ and $l$ add coherently.

In all forms of the stripping theory in use, the differential cross-section depends only on the orbital momentum $l$ of the captured nucleon, not its total, $j=l \pm 1 / 2$. The two $j$ values do not interfere, so we may write

$$
S_{l}=\Sigma_{j} \theta_{j i}{ }^{2} \text {. }
$$

Following French and $\operatorname{Raz}(2)$, the differential cross-section may then be written $^{\mathrm{I}}$

$$
\frac{d \sigma(\theta)}{d \omega}=\frac{2 I_{B}+1}{2 I_{A}+1} \Sigma_{l} S_{l} \phi_{l}(\theta)
$$

and $\phi_{l}(\theta)$ called the intrinsic single-particle cross-section. $S_{l}$ contains the information on nuclear structure. The form (6) is useful for comparing values of $S_{l}$ for different nuclear levels but the same $l$. Although $\phi_{l}$ is energy-and $Z$-dependent, we believe its variation is slow generally, so that little error is introduced by using, for example, the simple Butler theory to estimate any change. Then in such a comparison the uncertainites in the absolute magnitudes of the crosssections, due to potential scattering effects etc. (12), are largely eliminated (2).

Although the protion angular distribution (6) does not depend on the $j$ of the captured neutron but only on the $l$, this is not true of the proton polarization (18) or the angular correlation of the proton with any ensuing $\gamma$-rays (19). These give a measure of the relative amounts of the two possible $j$ values, $j=l \pm{ }_{1}^{1}$ ', taking part in the capture. The more detailed information obtained would seem to make the extra effort required for these experiments well worthwhile. The polarization depends intimately upon the nature of the scattering interactions with the incoming deuterons and outgoing protons, vanishing if these are neglected as in the simple Butler theory. The $p-\gamma$ correlation is to some extent affected by these interactions, but is not fundamentally dependent on them. So, to the extent that the interactions are not well known, the polarization measurement is a less reliable source of information than the $p-\gamma$ correlation. 


\section{EXCITATION OF VIBRATIONAL LEVELS}

It was suggested by Bohr (3) that one should observe nuclear energy levels corresponding to the excitation of quadrupole (or ellipsoidal) shape vibrations. Each of the quanta (phonons) would have energy $h \omega$, angular momentum $2 \hbar$, and even parity. Even-even nuclei would show energy spectra of the form $E=$ $N h \omega$, with ground state $\mathbf{0}+$, first excited state (one phonon) $2+$. The second level is triply degenerate, since the two phonons can couple to resultant spins 0,2 , or 4 . In practice this degeneracy will be lifted by perturbations, so we expect a close $0,2,4+$ triplet. The perturbation arises from coupling to the underlying shell structure: the disturbance that the slow change in shape of the potential well will produce on the shell model particle orbits (3).

There is evidence for this type of collective motion in even-even nuclei with $70 \lesssim A \lesssim 150(6)$. In even-odd nuclei, we have in addition the angular momentum $J$ of the odd nucleons which, with that due to any phonons present, $R$, can form various resultants, $\mathbf{I}=\mathbf{J}+\mathrm{R}$. Thus each member of the vibrational band will show additional fine structure.

We can conveniently write the wave functions as $|N R J, I M\rangle ; N$ is the number of phonons present. The lowest levels in even-even nuclei will have $J=0$. The phase convention adopted is given by the coupling order (16),

$$
|N R J, I M\rangle=\Sigma m|N R M-m\rangle|J m\rangle\langle R J M-m m \mid I M\rangle .
$$

$|N R \mu\rangle$ is the vibrational wave function, $|J m\rangle$ is the shell model state for the nucleons. The reduced width amplitudes are readily obtained by inserting the states (7) into the overlap integral (1). Let us denote the quantum numbers of the target nucleus $A$ by the suffix 1 , those of the residual $B$ by 2 . A result immediately obvious from (1) is that the vibrational states of $A$ and $B$ must be the same. That is, $N_{1}=N_{2}$ and $R_{1}=R_{2}$, otherwise the orthogonality of the vibrational states makes the overlap vanish. We find

$$
\begin{array}{r}
\theta_{j l}\left(N_{1} R_{1} J_{1}, N_{2} R_{2} J_{2}\right)=\sqrt{\left(2 I_{1}+1\right)\left(2 J_{2}+1\right)} W\left(I_{1} J_{1} I_{2} J_{2} ; R_{1} j\right) \\
\times(-)^{I_{1}+J_{2}-R_{1}-j} \delta\left(R_{1} R_{2}\right) \delta\left(N_{1} N_{2}\right) \theta_{j l}\left(J_{1} J_{2}\right),
\end{array}
$$

where $\delta(a b)$ is the Kronecker delta. The last factor, $\theta_{j l}\left(J_{1} J_{2}\right)$, is just the overlap of the shell model states, independent of the associated collective vibrations, and is discussed in refs. (2) and (19). For example, if the target has $(n-1)$ neutrons in an orbit with $j$, and the residual nucleus has $n$, it is just $\sqrt{n}$ times the corresponding fractional parentage coefficient (15). However, we are here interested in the effect of the vibrational motion; this contributes an angular momentum coupling factor, the Racah $W$ coefficient (1\%), to the reduced width.

In the case of practical interest, the target is in its ground state, hence $N_{1}=$ $R_{1}=0$ and nucleon capture can only lead to no-phonon levels in the residual 
nucleus, $\left(N_{2}=R_{2}=0\right)$. The reduced width for these transitions is just the shell model value,

$$
\theta_{j l}\left(00 I_{1}, 00 I_{2}\right)=\theta_{j l}\left(I_{1} I_{2}\right)
$$

However, the same perturbations we mentioned, which split the degenerate two phonon levels in even-even nuclei, will at the same time introduce admixtures into the zero-order wave functions (7). The interaction is such as to change $N$ by \pm 1 for each order of perturbation, and $R$ and $J$ by $0, \pm 1$, or $\pm 2(20)$. These admixtures allow violations of the simple selection rules given abover, allowing capture into one-phonon levels to first order in the perturbation, into two-phonon levels to second order, and so on. Calculation of the reduced width amplitudes then follows (3) and (4); the $\theta(\alpha \beta)$ are given by (8).

To make a crude estimate of their magnitudes we use the following argument. The amplitude of a state $\left|\alpha^{\prime}\right\rangle$ admixed to the zero order state $|\alpha\rangle$ is given by first order perturbation theory as $\left\langle\alpha^{\prime}\left|H^{\prime}\right| \alpha\right\rangle /\left(E_{\alpha}-E_{\alpha^{\prime}}\right)$, of order $\lambda$ say, where $H^{\prime}$ is the perturbation. The second order terms are then of order $\lambda^{2}$. But the energy shift in second order is $\Sigma_{\alpha^{\prime}}\left\langle\alpha^{\prime}\left|H^{\prime}\right| \alpha\right\rangle^{2} /\left(E_{\alpha}-E_{\alpha^{\prime}}\right)$, which is of order $\left(E_{\alpha}-E_{\alpha^{\prime}}\right) \lambda^{2}$. Now the splitting of the two-phonon triplet in even-even nuclei in the few cases observed is of order $100 \mathrm{kev}$, while $\left(E_{\alpha}-E_{\alpha^{\prime}}\right)$ is of order $1 \mathrm{Mev}$ (roughly the particle $J=2, J=0$ spacing). So,

$$
\left(E_{\alpha}-E_{\alpha^{\prime}}\right) \lambda^{2} \sim \lambda^{2} \mathrm{Mev} \sim 0.1 \mathrm{Mev} .
$$

Then the first order admixtures to the wave function have amplitudes $\sim \lambda \sim 1 / 3$, the second order $\sim \lambda^{2} \sim 1 / 10^{5}$. The reduced widths $\theta^{2}$ are, of course, proportional to the squares of these amplitudes, so the capture probability to a 'one-phonon' level is roughly 1/10 that to the 'no-phonon' ground state, and to 'two-phonon' levels is roughly $1 / 100$. These figures, of course, are very rough. We note, however, that they reach down to the intensity of 'background' processes such as compound nucleus formation, so more accurate estimates are probably of little value.

The argument above has to be modified in one important case. If there is an excited-state of the underlying shell structure close to one of the vibrational levels with the same total spin $I$, the mixing of their wave functions can be considerable and the reduced width of the vibrational level correspondingly enhanced. Such appears to be the case in $\mathrm{Cd}^{114}$, the one nucleus where the twophonon triplet has been clearly identified (21). Close to the $0+, 4+$ members

${ }^{4}$ The small contributions discussed in Appendix II would also allow transitions between states differing by one phonon.

5 These estimates are also consistent with the observed $\gamma$-ray branching ratios $(\gamma)$ from the 'two-phonon' $2+$ level in even-even nuclei to the ground and 'one-phonon' states, and the $M 1 / E 2$ mixt,ure in the latter transition. The branching ratio is (third order) ${ }^{2}$ in the perturbation, that is $\sim \lambda^{6}$, while the energy dependence of $E 2$ transitions favours the crossover by a factor $\sim(2 h \omega / 1 h \omega)^{5} \sim 30$. The ratio is generally found to be a few per cent. 
of the triplet there are two $2+$ levels, at $1212 \mathrm{kev}$ and $1368 \mathrm{kev}$. One of these has to be considered to arise from a particle excitation $(N=R=0, J=2)$. The closeness of these two levels makes perturbation theory inadequate; a more detailed calculation shows a strong intermingling of the two zero-order wave functions, leading to reduced widths for both levels comparable to that for the ground state. The proximity of the particle excitation also enhances somewhat the width of the first excited 'one-phonon' level, but the widths for the $0+$ and $4+$ levels remain of the order estimated above.

\section{EXCITATION OF ROTATIONAL LEVELS}

We have reason to believe (3) that a large group of nuclei are strongly deformed into an ellipsoidal shape, displaying, for example, large quadrupole moments. Then the potential well of the shell model is also nonspherical, and its deformation may greatly influence the individual nucleon orbits. But in addition we have variables describing the shape and its orientation, with the possibility of rotational motion as wcll as vibration about the equilibrium shape. The deformation (and hence the moment of inertia) is sufficiently large for these nuclei to rotate adiabatically. That is, their rotation is so slow that Coriolis forces, etc., are almost negligible and disturb very little the nucleon orbits in the potential well. Then the nuclear wave function $\psi$ can be written as a simple product

$$
\psi \sim \phi_{\mathrm{vib}} D X
$$

of a function $\phi_{\mathrm{vib}}$ describing the nuclear shape and its state of vibration (the so-called $\beta$ and $\gamma$ vibrations), a function $D$ describing the orientation of the shape, and a 'deformed shell model' wave function $X$ describing the nucleons' motion in the deformed potential well.

Generally the equilibrium shape of lowest energy possesses axial symmetry, and its orientation is described by the symmetric top eigenfunctions, $D_{K M}{ }^{(I)}\left(\theta_{i}\right)$. $I$ is the total nuclear angular momentum, $M$ its $z$-component, and $K$ its projection on the nuclear symmetry axis. The corresponding eigenvalues give the wellknown rotational energy spectrum

$$
E=E_{0}+\frac{h^{2}}{2 \mathscr{G}} I(I+1) .
$$

However, what really concerns us here is the structure of the generalised 'deformed shell model.' As the potential well is no longer spherically summetric, the orbital $l$ and total angular momentum $j$ of each nucleon are no longer constants of the motion. However, as long as axial symmetry is preserved, $\Omega$, the projection of $j$ on the symmetry axis, does remain a good quantum number for each orbit. The different $\Omega$, degenerate in the spherical limit, now have different 
energies (except that $\pm \Omega$ remain degenerate if, as we assume, the nuclear shape has reflection symmetry about the equatorial plane: that is, it is not pearshaped). The wave functions for these orbits have been calculated by scveral authors (22) for various potential wells. It would be one of the principal objectives of stripping experiments to test these wave functions.

Each single particle function can be expanded in terms of the spherical limit functions, that is, eigenfunctions of angular momentum. If $w$ is the parity, and $\alpha$ represents the other two quantum numbers needed to specify fully the orbital $\chi_{\Omega}$,

$$
\chi_{\Omega w \alpha}=\Sigma_{N j l} C_{N j l}^{\prime}(\Omega w \alpha) \psi_{N j l \Omega} .
$$

$N$ is the principal quantum number of the spherical well eigenfunctions $\psi$. As the deformation is allowed to go to zero, of course, all but one of the coefficients $c$ vanish. For finite deformations they have been calculated in refs. (2Q2), or may be estimated by perturbation theory (Appendix III) ${ }^{6}$. The complete shell model wave function we need is then a normalized, determinantal (for antisymmetry), product of the individual nucleon orbitals, whose total projection $\Omega$ on the symmetry axis is the sum of the individual projections $\Omega_{i}$ :

$$
X_{\Omega}=\operatorname{det} \chi_{\Omega_{i}}, \quad \Omega=\Sigma_{i} \Omega_{i} .
$$

The complete nuclear wave function (10) now takes the well-known form (3), symmetrized to be invariant under reflection in the equatorial plane.

$$
\Psi(I M K \Omega)=\sqrt{\frac{2 I+1}{16 \pi^{2}}} \phi_{\mathrm{vib}}\left\{X_{\Omega} D_{K, M}{ }^{(I)}+(-)^{P} X_{-\Omega} D_{-K M}{ }^{(I)}\right\} .
$$

The phase $p=I+K-\Omega-1 / 2 A . X_{-\Omega}$ is a determinant similar to $X_{\Omega}$ except that each constituent $\Omega_{i}$ has changed sign; we use the phase convention $c_{j}\left(-\Omega_{i}\right)=$ $(-)^{j-1 / 2} c_{j}\left(\Omega_{i}\right)$ for the coefficients in (12). The low-lying levels of interest to us have $K=\Omega$, and their vibrational state will be simply that of zero point oscillations. When $K=\Omega=0$, the symmetry of (14) demands $I$ be even only, and the normalization has to be readjusted,

$$
\Psi(I M 00)=\sqrt{\frac{2 I+1}{8 \pi^{2}}} \phi_{\mathrm{vib}} X_{0} D_{0 M}{ }^{(I)} .
$$

We are now in a position to calculate the reduced widths, using (1). The presence of rotation merely introduces a projection factor, representing the probability of finding the rotating target and incident nucleon in correct relative align-

6 In Nilssons' calculations (22), a representation different from (12) is used. His coefficients $a$ are related to ours by a Clebsch-Gordan transformation (16),

$$
c_{N j l}(\Omega w \alpha)=\Sigma_{\Lambda} a_{N i \Lambda}\langle l, / 2 \Lambda \Omega-\Lambda \mid j \Omega\rangle .
$$

(See Appendix IV.) 
ment for capture. Formally this arises because the particle motion is described in $\chi_{\Omega}$ in coordinates $x^{\prime}$ relative to the rotating nucleus and the expansion (1) is in a space-fixed coordinate system. The neutron function in (1) has to be expressed relative to body-fixed axes

$$
\phi_{j l m}\left(x_{N}\right)=\Sigma_{m^{\prime}} \phi_{j l m^{\prime}}\left(x_{N^{\prime}}\right) D_{m^{\prime} m}{ }^{(j)}\left(\theta_{i}\right) .
$$

The overlap integral is then readily evaluated with the wave functions (1+), using the relation

$$
\int d \theta_{i} D_{a \alpha}{ }^{(A)}\left(\theta_{i}\right)^{*} D_{3 \beta}{ }^{(B)}\left(\theta_{i}\right) D_{c \gamma}{ }^{(C)}\left(\theta_{i}\right)=\frac{8 \pi^{2}}{2 A+1}\langle A a \mid B C b c\rangle\langle A \alpha \mid B C \beta \gamma\rangle .
$$

We see immediately that for a nonzero reduced width or overlap, the initial and final shell model states $X_{\Omega}$ must not differ in more than one single nucleon orbital. The amplitude for capture with $j$ and $l$ is then proportional to the corresponding coefficient $c_{j l}$ in the expansion (12) of that extra orbital. Designating as before the target and residual nuclear quantum numbers by suffices 1 and 2 , respectively, we find

$$
\begin{aligned}
\theta_{j l}\left(K_{1} \Omega_{1}, K_{2} \Omega_{2}\right)=g \sqrt{\frac{2 I_{1}+1}{2 I_{2}+1}}\left\langle I_{2} K_{2}\right| I_{1} j \mp K_{1} \Omega_{2} & \left. \pm \Omega_{1}\right\rangle \\
& \times\left\langle\phi_{2} \mid \phi_{1}\right\rangle c_{, l}\left(\Omega_{2} \pm \Omega_{1}\right) .
\end{aligned}
$$

$g$ is $\sqrt{2}$ if either $K_{1}=\Omega_{1}=0$ or $K_{2}=\Omega_{2}=0$, and unity otherwise. The \pm is taken as the captured neutron enters an orbit with $\Omega=\left|\Omega_{2} \pm \Omega_{1}\right|$. The upper sign in practice might occur if the residual nucleus were odd-odd. The projection factor mentioned above appears as a Clebsch-Gordan coefficient (16). It provides the first selection rule: $K_{1} \pm K_{z}=\Omega_{1} \perp \Omega_{2}$, taking \pm as before. Since the target in general would have $K_{1}=\Omega_{1}$, this leads to $K_{2}=\Omega_{2}$. In addition, only $j \geq\left|\Omega_{2} \pm \Omega_{1}\right|$ is allowed, even if the values of $I_{1}$ and $I_{2}$ would seem to permit smaller values of $j$. We return to this below.

The factor $\left\langle\phi_{2} \mid \phi_{1}\right\rangle$ in (16) is the overlap of the initial and final vibrational states (zero point fluctuations in shape for low lying levels). The approximate forms for $\phi_{\mathrm{vi}}$ given by Bohr and Mottelson (3) may be used to estimate this overlap. For nuclei with little difference in deformation, $\left\langle\phi_{2} \mid \phi_{1}\right\rangle$ should depart little from unity; for example a $10 \%$ change in delormation may produce an overlap of 0.9 or 0.8 . However, it has an exponential dependence on the square of the difference in deformations, so could produce a strong reduction in capture probability if the nuclei had markedly different shapes ${ }^{7}$.

${ }^{7}$ When the deformations of the target and residual nuclei differ, the otherwise similar single nucleon orbits will not completely overlap. But again, it is easy to show that this is not serious unless the nuclear shapes differ considerably. Also, it is not clear how much of this effect may be already included in some average way in the vibrational overlap. 
The orthonormal properties of the Clebsch-Gordan coefficient in (16) allow us to deduce a sum rule. If the square of (16) is summed over all final states $I_{2}$ in a given rotational band which are reached by capture with $j, l$, we get

$$
\Sigma_{I_{2}}\left(2 I_{2}+1\right) \theta_{j l}{ }^{2}=g^{2}\left(2 I_{1}+1\right) c_{j l}(\Omega)^{2} .
$$

If we then sum the contributions from different $j$ and $l$ in the same band of levels, we can use the normalization condition $\Sigma_{j l} c_{j l}{ }^{2}=1$ for a particular orbit (12),

$$
\Sigma_{j l I_{2}}\left(2 I_{2}+1\right) \theta_{j l}{ }^{2}=g^{2}\left(2 I_{1}+1\right) .
$$

With a spin zero target, $I_{1}=0$ and $j=I_{2}$, this reduces to the sum rule given by Litherland and McManus (8)

$$
\Sigma_{j l} \theta_{j l}{ }^{2}(2 j+1)=2,
$$

where we still sum only over final states in a particular rotation band. Care must be taken over application of this rule in practice when more than one $l$ value contributes. As we have stressed above, following (6), uncertainties in the intrinsic single-particle cross-section $\phi_{l}(\theta)$ are reflected in uncertainties in the relative reduced widths for different $l$ values.

It is important to compare transitions to different members of the same rotational band. Each level in a band has the same internal shell structure, with the same $K$ and $\Omega$, differing only in the amount of rotational motion as a whole. In addition, the vibrational character of each is the same, so the vibrational overlap drops out in a comparison between members. There are two cases of most interest. The first is with the even-even target nucleus which has $I_{1}=K_{1}=\Omega_{1}=0$. A nucleon is captured with $j, l$ to form the even-odd residual nucleus in the various members of the rotational band with $I_{2}=\Omega_{2}, \Omega_{2}+1, \cdots$ etc. Clearly we must have $j=I_{2}$ to conserve total angular momentum. But the nucleon enters the same orbit (with $\Omega_{2}$ ) for all levels in the band. So capture into successive levels explores, one at a time, the $j=\Omega_{2}, \Omega_{2}+1, \cdots$ parts of the wave function (12) for that orbit. (16) reduces to

$$
\theta_{j l}\left(00, \Omega_{2} \Omega_{2}\right)=\sqrt{\frac{2}{2 j+1}} c_{j l}\left(\Omega_{2}\right) \delta\left(j I_{2}\right)\left\langle\phi_{2} \mid \phi_{1}\right\rangle .
$$

A similar situation exists for capture by even-odd targets. But now the nonzero target spin, $I_{1}=s_{1}=K_{1} \neq 0$, allows capture with a particular $j$ to feed more than one level of a rotational band in the residual nucleus. The capture probabilities to two such levels differ only in the projection factors. So we are lead to a simple branching ratio rule, similar to those for $\alpha^{-}, \beta$ - and $\gamma$-transitions (23),

$$
\frac{\theta_{j l}{ }^{\prime}}{\theta_{j l}}=\sqrt{\frac{2 I_{2}+1}{2 I_{2}{ }^{\prime}+1} \frac{\left\langle I_{2}{ }^{\prime} \Omega_{2} \mid I_{1} j \mp I_{1} \Omega_{2} \pm I_{1}\right\rangle}{\left\langle I_{2} \Omega_{2} \mid I_{1} j \mp I_{1} \Omega_{2} \pm I_{1}\right\rangle}}
$$


When the residual nucleus is even-even, we have for the ground state band $\Omega_{2}=K_{2}=0, I_{2}=0,2,4 \cdots$. The Clebsch-Gordan coefficients in (16), (18) then have simple explicit forms, given in Appendix IV. The fact that we must have $j \geq \Omega_{1}=I_{1}$ leads to an interesting selection rule. If $I_{1}$ is greater than $1 / 2$, conservation of total angular momentum would permit capture with $j<I_{1}$ into one or more of the excited states. Since a stripping angular distribution enhances the effect of lower $l$ values, such a violation of the selection rule $j \geq I_{1}$ imposed by the model would be easily detected, (see Appendix II, though). Such an experiment would be a close analogue of the tests of the spherical shell model proposed a few years ago (24).

As an example, suppose the target ground state were $5 / 2+$. The residual $0+$ ground state can, of course, only be reached by capture of a $d_{5 / 2}$ nucleon. But the restriction $j \geq \Omega_{1}=5 / 2$ forbids $s_{1 / 2}$ capture into the excited $2+$ rotational level, although it is allowed by conservation of total angular momentum. Any such $l=0$ transitions would be readily seen in the angular distribution, against the main $l=2$ component. There may be some evidence for this rule in the reaction $\mathrm{Mg}^{25}(d, p) \mathrm{Mg}^{26}$ (25). The ground state of $\mathrm{Mg}^{25}$ is $5 / 2+$, so this reaction would be the one just described if $\mathrm{Mg}^{26}$ displays a rotational spectrum. In fact, $l=0$ capture to the $2+$ first excited state is observed to be only $1 / 20$ as intense as $l=2$. The somewhat uncertain experimental data indicate the reduced width ratio of this group to that feeding the ground state is about 1 , while (18) gives the value about $1 / 3$. However, this indicates the need to collect more precise data in this region of the periodic table.

Any departure of the capture probabilities from the values given above, or any breakdown of the selection rules could be due to two causes. The first is the approximate nature of the independence of the collective rotation and vibration and the particle orbits in the potential well, expressed by the product form of the wave function (10) or (14). In particular there is the rotation-particle coupling (3) which can mix (to first order) some states with $K \pm 1, \Omega \pm 1$ into the main wave function (14). If there are excited states with these characteristics close in energy to the band being studied, such mixing could be appreciable. These effects have been studied in detail for the spectrum of $\mathrm{W}^{183}$ by Kerman (26). Rotation-vibration coupling (3) produces a small correction to the rotational energy spertrum of the form $\Delta E=E^{(2)} I^{2}(I+1)^{2}$. Associated with this will be small admixtures of excited vibrational states, with amplitudes of order $\sqrt{\Delta E / h \omega}$, where $h \omega$ is the excitation energy of the $\beta$ or $\gamma$ vibrations. Thus there will be corrections of the same order to the reduced width amplitudes, and the branching ratio (18). For the values appropriate to the rare earth nuclei (26), however, these are negligible.

A second cause of deviations from the predictions of the simple model would arise from residual interparticle forces. Just as in the spherical shell model, the effects of the inter-nucleon forces are not exhausted by the average potential 
well used to define the single particle orbits. The nucleon motion is not completely independent. Inelastic collisions do occur, giving rise to configuration mixing. This directly affects the structure of $X_{\Omega}$ : no longer is it a simple determinant, but rather a sum of such terms. The two-body forces are scalar in nature so all terms would have the same value of $\Omega$, but, to first order, could differ in the excitation of one or two nucleons. The success of the simple model for the rare earth nuclei indicates that any residual interations are by no means as important as the deformation of the potential well in determining the overall structure of $X_{\Omega}$. The raising of the degeneracy in $\Omega$ by the deformation seems to reduce the effect of such interactions. However, there is evidence that pairing energy shifts the relative positions of single particle levels when two neutrons are added to a nucleus (27). Analysis of the binding energies of nuclei around $\mathrm{Mg}$ also reveals pairing energies of as much as $4 \mathrm{Mev}$ (28). In addition, configuration mixing is adequate to explain the observed magnetic moments of deformed even-odd nuclei (29).

In either case, the wave functions (14) remain a satisfactory representation, except that now we shall have one or more small admixed components, as in (3). The reduced widths of course must be calculated in accordance with (4), where the $\theta(\alpha \beta)$ are now of the form (16).

In conclusion, we see that stripping is a direct way of investigating the validity of the collective model wave functions for rotating deformed nuclei. In particular, experiments with an even-even target provide a step-by-step analysis (17) of the wave function of the added nucleon, and the branching ratio (18) is useful to test whether the residual nuclear levels form part of a rotational band.

\section{THE O-K COLLECTIVE MODEL}

For completeness, we conclude with a discussion of the reduced widths using the representation chosen by Osborn and Klema (7) as a kinematical model for the empirical correlation of nuclear ground state properties. While formally closely related to the Bohr-Mottelson (3) picture, this model emphasises the description of the ground state wave function rather than the energy spectrum.

An cven-odd nucleus is said to consist of the odd single nucleon moving in a shell model orbit $\phi_{j l m}$, coupled to collective motion of the 'core' with angular momentum $\lambda, \psi_{\lambda \mu}$. The basic wave functions are then

$$
\Psi(\lambda j l, I M)=\Sigma_{m} \phi_{g_{l} m} \psi_{\lambda_{M-m}}\langle\lambda j M-m m \mid I M\rangle .
$$

The nuclear wave function is then a certain mixture of these states.

$$
\Psi(I M)=\Sigma_{\lambda_{j l} l} a_{\lambda j l} \Psi(\lambda j l, I M) .
$$

To avoid introducing too many parameters, when fitting the ground state data Osborn and Klema assumed that two components of (20) were dominant.

These authors regard the collective motion of the core as that of an axially 
symmetric rigid rotator, with zero projection of the angular momentum $\lambda$ on the symmetry axis. $\psi_{\lambda_{\mu}}$ then becomes a spherical harmonic $Y_{\lambda_{\mu}}\left(\theta_{i}\right)$ in the polar angles of the core axis. Although this is a natural interpretation for nuclei where rotational energy levels are observed $(30)$, in other regions of the periodic table it might be more appropriate to take some other picture. A glance at (7) shows the wave function (19) (apart from a change in notation) has a form identical to that of the phonon model, so that the core motion could be of a vibrational type.

The equivalence of (19) and (7) shows that the expression for the reduced widths, and the associated selection rules, is the same as for the phonon model, (8) and (9). The stripping reaction then provides an independent check on the values of the coefficients $a_{\lambda j l}$ in (20) deduced from magnetic moments, ete. The neighbouring even-even nuclei are assumed to have ground states described simply by the core function $\psi_{I M}$, with $I=M=0$. Then the $\Delta \lambda=0$ selection rule shows that reactions leading to even-odd residual nuclei with ground states principally $\lambda \geq 2$ should be strongly inhibited compared to those with large $\lambda=0$ components. In these cases the reaction amplitude is just proportional to $a_{0 j l}$.

\section{APPENIIX I}

As mentioned in the introduction, the stripping of projectiles other than deuterons involves the same nuclear overlap factor (1). In addition, inelastic nucleon scattering of the type $A\left(p, p^{\prime}\right) B$, when considered to occur by the direct interaction of the incident nucleon with one in the target, requires a similar overlap for its description (1). This can be expanded analogously to (1),

$$
\begin{aligned}
\int d \xi \Psi_{B} *\left(x^{\prime} \xi\right) \Psi_{A}(x \xi)= & \Sigma_{P M j l j^{\prime} l^{\prime}} \beta_{j l}(P A) \beta_{j^{\prime} l^{\prime}}(P B)^{*} \phi_{j l M_{A}-M}(x) \\
& \times \phi_{j^{\prime} l^{\prime} M_{B}-M}\left(x^{\prime}\right)^{*}\left\langle I_{P} j M M_{A}-M \mid I_{A} M_{A}\right\rangle \\
& \times\left\langle I_{P} j^{\prime} M M_{B}-M \mid I_{B} M_{B}\right\rangle .
\end{aligned}
$$

For the direct amplitude, $x-x^{\prime}$ and represents the coordinates of the target nucleon responsible for the scattering. In the exchange terms, $x$ and $x^{\prime}$ are the coordinates of the ejected and captured nucleon, respectively. $P$ represents the various parent states obtained by removing the nucleon from $A$ or $B$; only parents common to both $A$ and $B$ can contribute. The parameters $\beta_{j l}$ are just the same as those in (1) which we have studied, with the parent $P$ playing the role of target nucleus.

The interpretation of inelastic scattering from nuclei exhibiting collective motion is complicated by the possibjilty of excitation through direct coupling of the incident nucleon to the collective modes (31). However, the two types of excitation may display different selection rules. For the excitation of vibrational 
levels, the direct process described above obeys the same rules as stripping: no change in vibrational state between $A$ and $B$. However, this rule is necessarily violated by the surface coupling; in Born approximation only states differing by one phonon are connected. Both processes can excite a ground state rotational band, but in Born approximation the surface coupling does not reach particle excitations. Of course, surface coupling does not compete in the analogous $(p, n)$ or $(n, p)$ reactions.

\section{APPENDIX II}

The coupling which is present in a $(d, p)$ reaction between the incident nuclcons and the collective modes of vibration or rotation of the target may give additional small contributions to the reaction amplitude which could be important when discussing the violation of the simple selection rules given above. To study this we use the exact form of the reaction amplitude given by Gerjuoy (32). This is proportional to

$$
\left\langle\psi_{f}\left|V_{N P}+V_{P T}\right| \Psi\right\rangle .
$$

$\psi_{f}$ described the residual nucleus, and protons in a plane wave state. $V_{N P}$, the dominant term, is the potential binding the deuteron, and $V_{P T}$ is the interaction with the target of the proton in the incident deuteron. $\Psi$ is the total wave function; the Born approximation consists of replacing it by $\psi_{i}$, which describes just the target nucleus and incident deuteron beam. Now $V_{P T}$ includes terms not scalar in the proton coordinates and thus coupling the proton to the collective motion of the target. In the simplest approximation, regarding $V_{T T}$ as an oscillating potential well, these are the well-known surface coupling terms of ref. 3 . It has been estimated that at medium energy the effect of $V_{P T}$ is at most about $10 \%$ of $V_{P N}(33)$, and the surface coupling term will be only a small part of this (of order $k \beta / U_{0}$ for rotational levels, $\left(k / U_{0}\right) \sqrt{h \omega / 2 C}$ for phonon states (3), if $U_{0}$ is the shell model potential well depth). However, its possible importance lies in its principally quadrupole nature, that is, because it behaves like a spherical harmonic $Y_{2 \mu}$ in the space of the captured neutron. So that if the neutron is captured with orbital momentum $l$, terms appear in the angular distribution which are characteristic of $l \pm 2$ captures, if allowed by conservation of total angular momentum. (Not $l \pm 1$ because of parity). Since lower $l$-values have greater intrinsic cross-sections, it would be possible for such an $l-2$ contribution to be non-negligible in comparison with the main $l$ term arising from $V_{N P}$. This is clearly important if we are investigating the validity of the selection rules discussed in the main text, which demand capture with $l$, even though conservation of total angular momentum would seem to allow $l-2$. Quite small admixtures of $l-2$ can be seen experimentally; for example, a few per cent of $s$-wave in a predominantly $d$-wave capture (24).

Sawicki (34) has pointed out that similar $l-2$ terms appear if distorted, not 
plane, waves are used for the deuterons and protons (12) and the distorting potential is nonspherical; even without such distortion, the approximation of evaluating the stripping matrix elcment at or near the nuclear surface $r=R(11)$ introduces these small contributions when the surface is nonspherical.

\section{APPENDIX III}

In many cases perturbation theory may be used to estimate the coefficients of the expansion (12) of the wave function for a nucleon orbit in a nonspherical potential well. Indeed, in view of the uncertainties in the exact radial dependence of the potential well, the way it is deformed, the effect of the deformation on the spin-orbit coupling (29), and so on, such a calculation may be as reliable (if not as convenient) as an exact solution for a particular choice of well. For axially symmetric nuclei with a quadrupole distortion we can use the potential

$$
U(\mathbf{r})=U_{0}(r)-k(r) \beta Y_{20}(\theta \phi) .
$$

If the spherical well $U_{0}$ is square, $k$ has the form of a delta function at the surface for small deformations. The deformation parameter $\beta$ is related to the intrinsic quadrupole moment (B),

$$
Q_{0}=\frac{3}{\sqrt{5 \pi}} Z R^{2} \beta
$$

Any spin-orbit coupling is included in the spherical well $U_{0}$, which defines the states $\psi_{N j l \Omega}$ in the expansion (12). Consider the orbit which in the spherical limit $\beta=0$ becomes $\psi_{N j l \Omega}$. In the usual way, the coefficients in (12) are, to first order in the perturbation,

$$
c_{N^{\prime} j^{\prime} l^{\prime}}(\Omega)=\beta\left\langle N^{\prime} l^{\prime}|k| N l\right\rangle\left\langle j^{\prime} \Omega\left|Y_{20}\right| j \Omega\right\rangle / \Delta E .
$$

$\Delta E$ is the separation of the unperturbed states in the spherical well, $\Delta E=$ $E\left(N^{\prime} j^{\prime} l^{\prime}\right)-E(N j l)$. The matrix elements of $k$ are roughly independent of the orbits involved; they have been estimated ( 3 ) to be about $40 \mathrm{Mev}$. The spherical harmonic matrix element is independent of $l, l^{\prime}$ provided they have the same parity.

$$
\begin{array}{ll}
\left\langle j^{\prime} \Omega\left|Y_{20}\right| j \Omega\right\rangle=\left\langle j \Omega\left|Y_{20}\right| j^{\prime} \Omega\right\rangle & \\
=\sqrt{\frac{5}{64 \pi}} \frac{3 \sqrt{(j-\Omega+1)(j-\Omega+2)(j+\Omega+1)(j+\Omega+2)}}{2(j+1)(j+2)}, & j^{\prime}=j+2 ; \\
= & -\sqrt{\frac{5}{64 \pi} \frac{3 \Omega \sqrt{(j-\Omega+1)(j+\Omega+1)}}{j(j+1)(j+2)},} \quad j^{\prime}=j+1 ; \\
=-\sqrt{\frac{5}{64 \pi} \frac{3 \Omega^{2}-j(j+1)}{j(j+1)},} & j^{\prime}=j .
\end{array}
$$

If the deformation strongly couples two orbits because their unperturbed energies 
are close, that is, $\Delta E$ in (22) is small, the perturbation may be exactly diagonalized in these two states, the others still being treated approximately as in (22). In this way we can obtain a very good idea of the structure of the single particle wave function $\chi_{\Omega}$ without making very detailed assumptions about the potential well, or calculating the exact solution.

\section{APPENDIX IV}

The Clebsch-Gordan coefficients, relating the coefficients of Nilssons' calculations $^{6}(22)$ to those we use, have a simple explicit form.

$$
\begin{gathered}
\Omega-\Lambda=1 / 2 l_{2} \Lambda \Omega-\Lambda|j \Omega\rangle \\
j=l+12: \sqrt{\frac{j+\Omega}{2 j}} \quad \sqrt{\frac{j-\Lambda}{2 j}} \\
j=l-1 / 2: \sqrt{\frac{j-\Omega+1}{2 j+2}} \quad \sqrt{\frac{j+\Omega+1}{2 j+2}} .
\end{gathered}
$$

The reduced width for capture of nucleons by an even-odd nucleus to form the even-even residual nucleus in a rotation state (16) includes the Clebsch-Gordan coefficient $\left\langle I_{2} 0 \mid I_{1} j I_{1}-I_{1}\right\rangle$. For the first three levels of the band, $I_{2}=0,2,4$, this has the form

$$
\begin{aligned}
& I_{2}=0 \quad j=I_{1}: \\
& \frac{1}{\sqrt{ } 2 \overline{j+1}} \\
& I_{2}=2 \quad j=I_{1}: \\
& \sqrt{\frac{5 j(2 j-1)}{(j+1)(2 j+1)(2 j+3)}}, \\
& j=I_{1}+1: \\
& \sqrt{\frac{15(j-1)}{j(j+1)(2 j+1)}}, \\
& j=I_{1}+2 \text { : } \\
& \sqrt{\frac{30}{j(2 j-1)(2 j+1)}} ; \\
& I_{2}=4 \quad j=I_{1}: \\
& \sqrt{\frac{9 j(j-1)(2 j-1)(2 j-3)}{(j+1)(j+2)(2 j+1)(2 j+3)(2 j+5)}}, \\
& j=I_{1}+1 \text { : } \\
& \sqrt{\frac{90(j-1)(j-2)(2 j}{j(j+1)(j+2)(2 j+1)(2 j+3)}}, \\
& j=I_{1}+2 \text { : } \\
& \sqrt{\frac{810(j-2)(2 j-5)}{j(j+1)(2 j-1)(2 j+1)(2 j+3)}}, \\
& j=I_{1}+3: \\
& \sqrt{\frac{1890(j-3)}{j(j+1)(j-1)(2 j+1)(2 j-1)}}, \\
& j=I_{1}+4: \\
& \sqrt{\frac{3780}{j(j-1)(2 j-3)(2 j-1)(2 j+1)}} .
\end{aligned}
$$

ReCeIved: October 30, 1957 


\section{REFERENCES}

1. R. Hubr, Progress in Nuclear Physics 3, 177 (1953); S. T. Butler, Phys. Rev. 106, 272 (1957).

2. A. M. Lane, Proc. Phys. Soc. A66, 977 (1953); J. B. French and B. J. Raz, Phys. Rer. 104, 1411 (1956); O. M. Bilaniuk and P. V. C. Hotgh, Phys. Rev. (to be published).

3. A. Bohr ani B. R. Mottelson, Kgl. Danske Videnskab. Selskab. Mat.fys. Medd. 27, No. 16 (1953); and in "Beta and Gamma Rayy Spectroscopy" (K. Sieghahn, ed.), p. 468. North-Holland, Amsterdam, 1955.

4. A. F. Litherland, E. B. Pavl, G. A. Bartholomew and H. E. Gove, Phys. Rev. 102, $208(1956)$.

5. E. B. PACL, Phil. May. 2, 311 (1957).

6. G. Scharfy-Goldhaber and J. Weneser, Phys. Rev. 98, 212 (1955).

7. R. K. (OsBorn anis E. D). Klema, Nuclear Physics 2, 454 (1957).

8. D. A. Bromley, H. E. Gove, and A. E. Litherland, Can. J. Phys. 35, 1057 (1957); A. F. Litherland, A. McMants, E. B. Paul, 1). A. Bromley, axi) H, L. Giove (to be published).

9. S. Yoshida, Progr. Theoret. Phys. (Japan) 12, 141 (1954); G. R. Sa tchler, Phys. Rev. 97, $1416(1955)$.

10. S. T. Bretler, Proc. Roy. Soc. A208, 559 (1951).

11. A. B. Bhatia, K. Huang, R. Huby, axis H. C. Newns, Phil. Mag. 43, 485 (1952).

12. W. Tobocman and M. M. Kalos, Phys. Rev. 97, 132 (1955).

13. A. P. Frexich, Phys. Rev. (to be published).

14. G. Ji. OWen and L. Madansky, Phys. Rev. 105, 1766 (1957).

15. A. M. lane and 1). H. Wrlkinson, Phys. Rev. 97, 1199 (1955).

16. W. U. Condon AND G. H. Shorthey, "The Theory of Atomic Spectra." p.73. Cambridge Univ. Press, London and New York, 1935.

17. A. Simon, J. H. Vander-Sluis, axid L. C. Biedenharn, "Tables of Racah Coefficients," ()ak Ridge National Laboratory Report. ()RNL-1679, 1954.

18. J. Horowitz and A. M. L. Messiah, J. phys, radium 14, 731 (1953); W. Cheston, Phys, Rev. 96, 1590 (1954).

19. G. R. Satchler, Proc. Phys. Soc. A67, 471 (1954).

20. 1). C. Chotmhury, Kgl. Danske Videnskab. Selskab, Mat.flys. Medd. 28, No. 4 (1954).

21. H. Motz, Phys. Rev. 104, 1363 (1956).

22. B. G. Nilsson, Kgl. Danske Videnskab. Selskah, Mat.fys. Medd. 29, No. 16 (1955); B. R. Mottelson and S. G. Nilsson, Phys. Reu. 99, 1615 (1955); S. A. Moszkowksi, Phys. Rev. 99, 803 (1955); K. Gottfried, Phys. Rev. 103, 1017 (1956).

23. G. Alaga, K. Alder, A. Bohr, And B. R. Mottelson, Kgl. Danske Videnskab. Selskab, Mat.fys. Medd. 29, Nio. 9 (1955).

24. W. C. Parkinson, E. H. Beach, and J. S. King, Phys. Rev. 87, 387 (1952).

25. J. R. Holt and T. N. Marsham, Proc. Phys. Soc. A66, 258 (1953).

26. A. K. Kerman, Kgl. Danske Videnskab. Selskab, Mal.fys. Medd. 30, No. 15 (1956).

27. D. Chase, unpublished calculations (1956).

28. D. M. Brinh and A. K. Kerman, private communication (1957).

29. J. N. L. Gacvis, thesis, Oxford University (1957).

30. E. D. Klema and R. K. (Osbonn, Nuclear Physics 3, 571 (1957).

31. 1). M. Brink, Proc. Phys. Sor. A68, 994 (1955); F. V. Inopis, J. Exptl. Theoret. Phys. (U.S.S.R.) 31, 901 (1956); Soviet JE'TP 4, 764 (1957).

32. E. Gerutoy, Phys. Rev 91, 645 (1953).

39. G. B. OWEN AND T. FlLTON, unpublished calculations (1957).

34. J. SAwICKI, private communication (1957). 